\title{
Pan-sharpening based on Merged Product of Spatial Frequency Components of PAN and Intensity Images
}

\author{
Megha K. Mehta \\ P.G. Scholar \\ SVIT, VASAD, India
}

\author{
Paru Thakkar \\ Project Manager \\ BISAG, Gandhinagar, India
}

\author{
Madhukar B. Potdar, Ph.D \\ Project Director \\ BISAG, Ghandhinagar, India
}

\begin{abstract}
Image fusion or Pan Sharpening produces high quality fused multispectral image using high spatial resolution Panchromatic (PAN) image and low spatial resolution multispectral (MS) image. The aim is to retain to a large extent both the high frequency spatial component of PAN and high spectral resolution of MS data. There are many approaches (more than 10) for image fusion based on different colour models and spatial transformations, the performances of some of which using the IKONOS-2 PAN and MS images have been evaluated earlier by the authors of this paper. In this paper, the concept of combining different spatial frequency components of the PAN and intensity images and merging with hue and saturation components is attempted on all the 10 approaches of image fusion. The technique of merging of high spatial frequency of PAN and low spatial frequency component of intensity images is described. The results of the statistical analysis show that there is marked improvement in retention of the spectral information albeit at slight loss of spatial correlation in pansharpening. The high level of retention of spectral information is especially significant in view of expected better classification accuracy.
\end{abstract}

\section{Keywords}

Image fusion, pan sharpening, spectral merging, spatial merging, IKONOS-2, image filtering.

\section{INTRODUCTION}

The sensors on board remote sensing satellites like IRS, IKONOS-2, Orbview, Quickbird etc., give two types of images, one is Multispectral image (MS) and another one is Panchromatic (PAN) image. MS sensors have coarse spatial resolution (e.g. $4 \mathrm{~m}$ for IKONOS-2) and fine spectral resolution while PAN sensors have fine spatial resolution (1 $\mathrm{m}$ for IKONOS-2) and coarse spectral resolution [1-5]. These are controlled by the signal-to-noise $(\mathrm{S} / \mathrm{N})$ ratio attainable for precision of radiance measurements by the sensors in use. Ideally, the resource scientist and technologist prefer the image with both high spectral and spatial resolution comparable to those of MS and PAN data, respectively. One way is to design sensors which can achieve $\mathrm{S} / \mathrm{N}$ ratio ideal for high spectral resolution (like MS images) while achieving high spatial resolution (like PAN data). Such a sensor system puts lot of load in terms of data acquisition, telemetry, data pre-processing and hence in huge cost. Pan-sharpening is a way out of these problems to compose images with high spectral and spatial resolutions from MS and Pan data as acquired from the prevalent satellite sensor systems.

Various algorithms for image fusion have been described in literature [2-11]. They are based on different colour models. In these algorithms using forward transformation Intensity (I), Hue $(\mathrm{H})$ and saturation $(\mathrm{S})$ are obtained using Red, Green and
Blue plane of MS image. Then Intensity (I) component is histogram matched with PAN image. These histogram matched image is replaced with intensity in inverse transformation process. Histogram matching will help to minimize distortion produced due to mismatching spectral response of PAN and MS of IKONOS-2 sensor. But, with this method over-injection of low-spatial-resolution of MS image will arise [6]. To overcome effect of over-injection the new algorithm is proposed in this paper. Dhamecha et al. [2,3] have suggested new approach to fusion and controlled injection (depending on the vegetation vigour and coverage) of high frequency component of Pan to obtain improved retention of spatial and spectral information.

The technique of image fusion, also known as spectral merging and pan-sharpening, of combining the spatial information content in pan data and spectral information in MS data is a smart way of achieving both high spatial and spectral resolutions at low cost, albeit at some loss or distortion in spectral information. The fusion methods developed are based on various colour models and spatial transformations. In the earlier work [1], the authors have evaluated the performance of various colour models suggested in conjunction with spatial transformations. The performances were evaluated in terms of statistical parameters such spatial correlation (sCC), spectral correlation (CC), difference index (DI), change in parameters such as entropy, normalized RMSE, S/N ratio etc. In these methods, the intensity component obtained by transforming red, green and blue spectral images to intensity, hue and saturation components following various colour models and spatial transformations.

Here, the same study is extended by following a modified approach to fusion. This paper describes the process of Image fusion/pan-sharpening using the new concept of frequency component injection. Using this concept some algorithms based on different colour models have been applied on a sample of IKONOS-2 pan and multispectral data. The performances of various algorithms are evaluated through different statistical parameters of fusion mainly keeping in view the spectral and spatial correlations.

\subsection{Data-Set Used}

The IKONOS-2 satellite Multi-spectral and Panchromatic data are used. IKONOS-2 was launched on September 24, 1999, and provides imagery since January 1,2000 . IKONOS-2 data downloaded from the NASA/GLCF web site are used to study land use types. The IKONOS-2 satellite data have MS resolution of $4 \mathrm{~m}$ and PAN resolution of $1 \mathrm{~m}$. Spectral range of blue band is $455-520 \mu \mathrm{m}$, green band is $510-600 \mu \mathrm{m}$, red band is $630-700 \mu \mathrm{m}$, NIR band is $760-850 \mu \mathrm{m}$ and, PAN band is $760-850 \mu \mathrm{m}$. The swath coverage is $11 \mathrm{~km}$. One scene $4 \mathrm{~m}$ resolution MS data of size about 2750x2750 pixels were resampled to size of $11000 \times 11000$ to match to the $1 \mathrm{~m}$ resolution of PAN image. The resultant images of both MS 
and PAN are then subset into multiple scenes of $512 \times 512$ pixels. Out of these, five subsets were chosen such that they covered wide range of land use classes such as soil, vegetation, wasteland, and urban.

\section{METHODOLOGY}

The methodology followed is shown in the flow chart (Fig. 1). Specifically, the Red, Green and, Blue components of MS data and pan data are spatially co-registered as the two sensors data are acquired synchronously from the same platform. All the data are brought to same radiometric range by histogram stretching from 0 to 255 ranges. The Intensity, Hue and Saturation (IHS) forward transformations based on various colour models and spatial transformations given by [3] are applied to generate the Intensity (I), Hue (H) and, Saturation (S) components. Usually, in all the methods, the intensity component is replaced with Pan image after matching its histogram with that of the intensity image. In deviation, the present methodology uses high pass filtered PAN merged with the low pass filtered intensity to generate new intensity component to be used in reverse transformation. The kernel

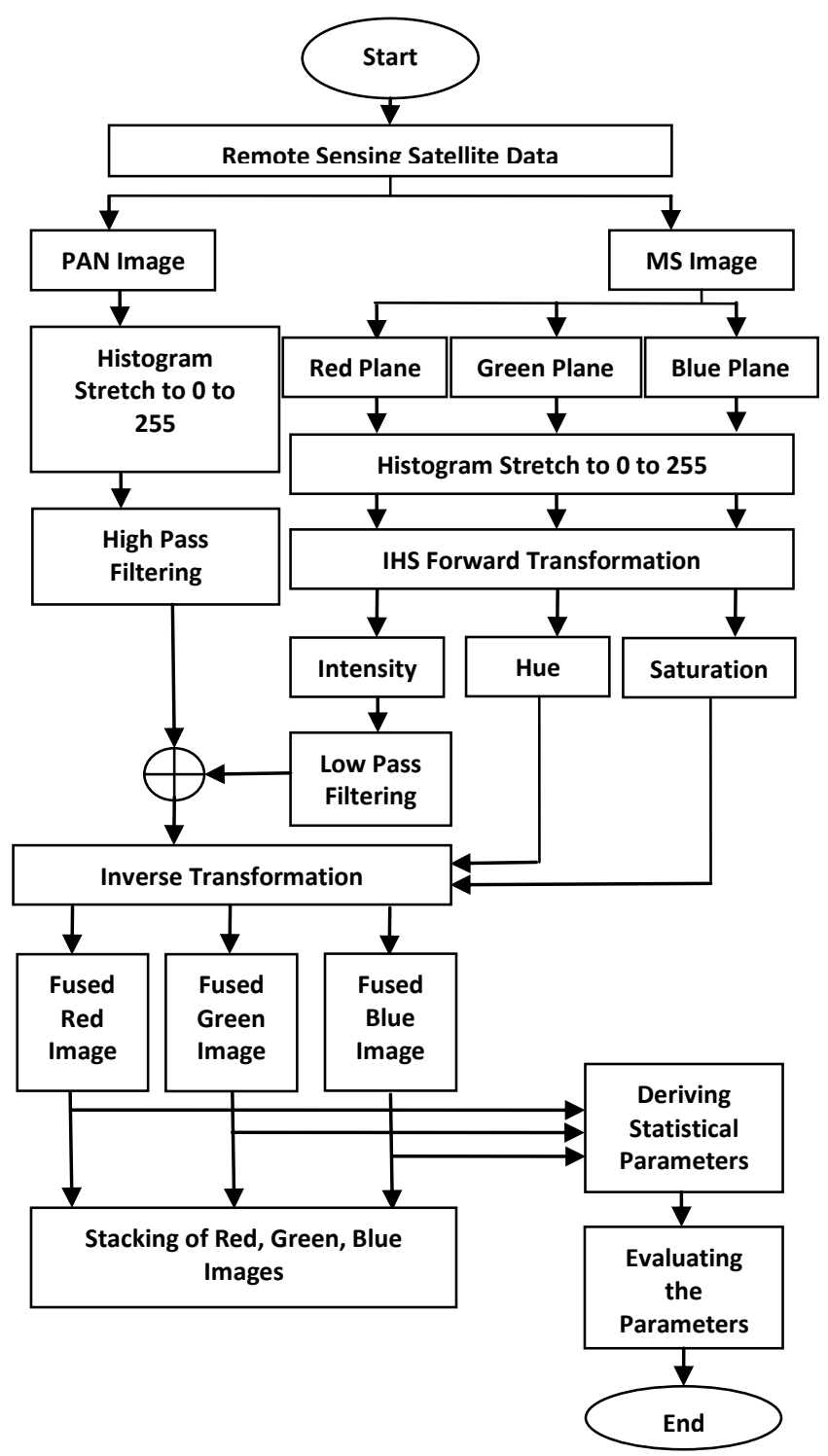

Fig.1. Flow Diagram for Pan-sharpening of IKONOS-2 data based approach for filtering of images is followed. Odd kernels of size ranging from 3 to 15 are used. With increasing kernel size, the higher spatial frequencies are filtered out. Then, the high pass filtered PAN and low pass filtered intensity are added to generate new intensity component which is expected to contain high spatial frequency (high resolution) information from pan data and high spectral information as well as low spatial resolution from MS data. After inverse transformation using new intensity image and hue and saturation images from forward transformation, the fused MS images are generated. The resultant fused MS images are then subjected to statistical analysis using parameters like SNR (signal to noise ratio), CC (correlation coefficient), sCC (spatial correlation coefficient), DI (difference index) to evaluate quality of fused image.

The Kernels of various sizes ranging from 3 to 15 for filtering are designed in such a way that when the high pass and low pass images are added, the original image is retrieved. The kernels for low and high pass filtering are as given below:

$$
\begin{gathered}
3 \times 3 \text { Kernel (low pass filter) }=\frac{1}{8}\left[\begin{array}{ccc}
1 & 1 & 1 \\
1 & 0 & 1 \\
1 & 1 & 1
\end{array}\right] \\
3 \times 3 \text { Kernel (high pass filter) }=\frac{1}{9}\left[\begin{array}{ccc}
-1 & -1 & -1 \\
-1 & 9 & -1 \\
-1 & -1 & -1
\end{array}\right]
\end{gathered}
$$

When these two kernels are added, the result will be near about null kernel. The Pan image data and the intensity component from HIS transformation are subjected to both high pass and low pass filtering. The high pass component of Pan and low pass component of intensity image then combined to obtain new intensity component to be used in inverse transformation which yields fused Red, Green and Blue components.

\section{RESULTS AND DISCUSSION}

In paper [3], various fusion algorithms are described based on different colour transformations and in some cases involve certain radiometric and spatial transformations. The algorithms have been named as HSV, ISH1, ISH2, ISH3, ISH4, ISH5, ISH6, ISH7, and HLS. Here the I stands for intensity, H stands for Hue, S for Saturation, V for Value, and L for Luminance. In this study, the data used are the same as those used in paper [1] for investigation. For the process of image fusion, injection of PAN image is used after histogram matching with Intensity component. There are problems of over-injection. To overcome this problem, instead of using whole frequency of MS and PAN images, the frequency components obtained after high pass filtering of PAN image and low pass filtering of intensity component (MS image) are used for fusion improvement can be achieved.

The IKONOS-2 data set is fused using different fusion algorithms described in $[4,5,9,10$, and 13]. Software implementation of fusion process as well as statistical parameters are done using MATLAB tool. Here, original PAN image and intensity component (MS image) are used after filtering with high pass filter and low pass filter respectively. With help of this process high frequency component of PAN is injected and fusion of MS and PAN is done. 


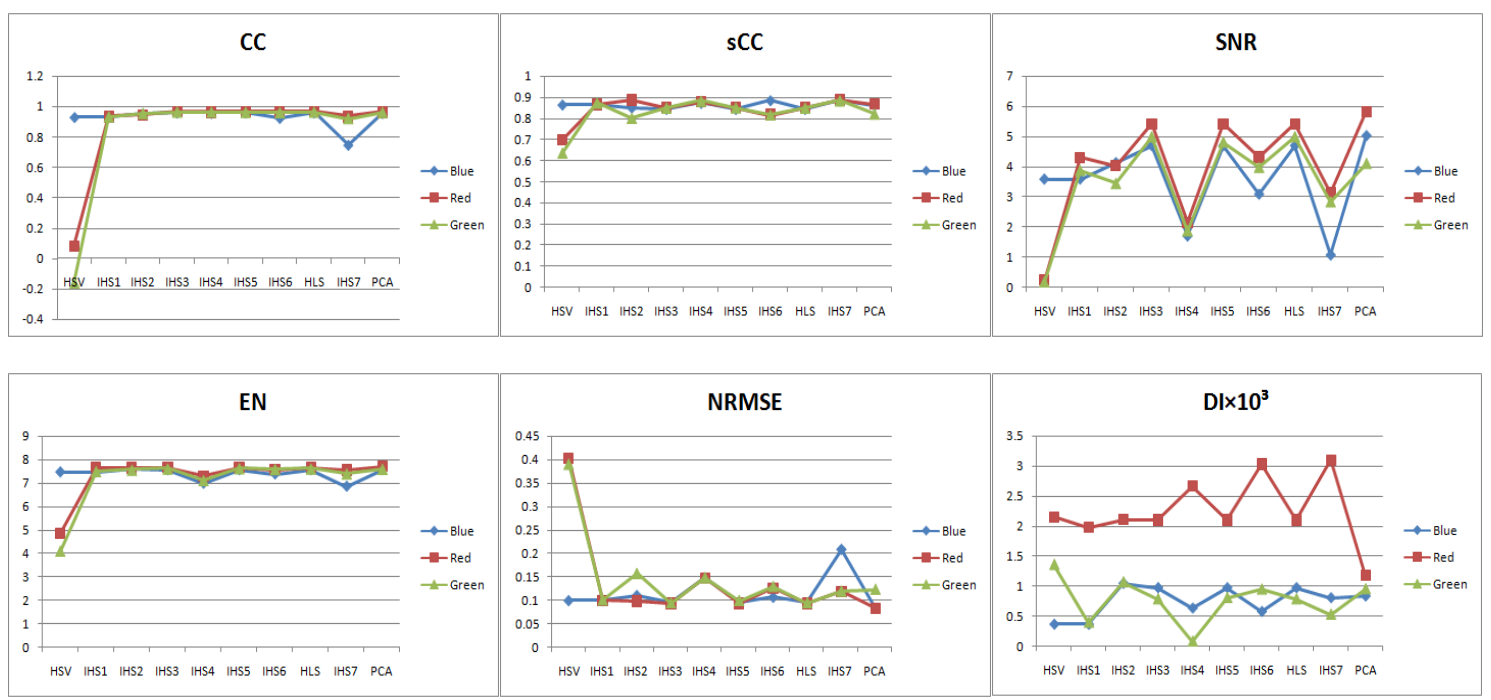

Fig.2. Variation of various statistical parameters depending on colour model and spatial transformations

Table 1: Statistical parameters of fusion following different

methods

\begin{tabular}{|c|c|c|c|c|c|c|c|c|}
\hline Method & Band & SNR & NRMSE & EN & DI×10 & CC & SD & SCC \\
\hline HSV & R & 0.246 & 0.402 & 4.866 & 2.149 & 0.084 & 18.660 & 0.697 \\
\hline & G & 0.195 & 0.391 & 4.114 & 1.364 & -0.158 & 15.594 & 0.634 \\
\hline & B & 3.584 & 0.099 & 7.462 & 0.371 & 0.930 & 48.116 & 0.863 \\
\hline IHS1 & R & 4.296 & 0.099 & 7.669 & 1.978 & 0.934 & 54.833 & 0.865 \\
\hline & G & 3.883 & 0.099 & 7.507 & 0.400 & 0.931 & 49.855 & 0.872 \\
\hline & $\mathrm{B}$ & 3.584 & 0.099 & 7.462 & 0.371 & 0.930 & 48.116 & 0.863 \\
\hline IHS2 & R & 4.025 & 0.099 & 7.630 & 2.106 & 0.942 & 56.174 & 0.886 \\
\hline & G & 3.451 & 0.157 & 7.566 & 1.074 & 0.953 & 73.237 & 0.801 \\
\hline & $\mathrm{B}$ & 4.124 & 0.111 & 7.601 & 1.049 & 0.952 & 65.529 & 0.849 \\
\hline IHS3 & R & 5.417 & 0.094 & 7.664 & 2.097 & 0.965 & 69.633 & 0.850 \\
\hline & $\mathrm{G}$ & 4.995 & 0.094 & 7.617 & 0.791 & 0.962 & 66.465 & 0.849 \\
\hline & $\mathrm{B}$ & 4.685 & 0.095 & 7.554 & 0.978 & 0.960 & 65.085 & 0.842 \\
\hline IHS4 & $\mathrm{R}$ & 2.147 & 0.148 & 7.296 & 2.662 & 0.963 & 44.638 & 0.877 \\
\hline & $\mathrm{G}$ & 1.888 & 0.148 & 7.125 & 0.084 & 0.962 & 40.833 & 0.882 \\
\hline & $\mathrm{B}$ & 1.712 & 0.148 & 6.977 & 0.640 & 0.957 & 40.202 & 0.871 \\
\hline IHS5 & $\mathrm{R}$ & 5.417 & 0.094 & 7.663 & 2.097 & 0.965 & 69.633 & 0.850 \\
\hline & $\mathrm{G}$ & 4.819 & 0.099 & 7.632 & 0.812 & 0.960 & 66.740 & 0.850 \\
\hline & $\mathrm{B}$ & 4.685 & 0.095 & 7.553 & 0.978 & 0.960 & 65.085 & 0.842 \\
\hline IHS6 & $\mathrm{R}$ & 4.314 & 0.127 & 7.564 & 3.031 & 0.963 & 73.592 & 0.816 \\
\hline & $\mathrm{G}$ & 3.981 & 0.129 & 7.598 & 0.952 & 0.959 & 70.917 & 0.816 \\
\hline & $\mathrm{B}$ & 3.088 & 0.107 & 7.374 & 0.583 & 0.924 & 49.196 & 0.884 \\
\hline HLS & $\mathrm{R}$ & 5.417 & 0.094 & 7.664 & 2.097 & 0.965 & 69.633 & 0.850 \\
\hline & $\mathrm{G}$ & 4.995 & 0.094 & 7.617 & 0.791 & 0.962 & 66.465 & 0.849 \\
\hline & $\mathrm{B}$ & 4.685 & 0.095 & 7.554 & 0.978 & 0.960 & 65.085 & 0.842 \\
\hline IHS7 & $\mathrm{R}$ & 3.132 & 0.119 & 7.535 & 3.096 & 0.932 & 51.988 & 0.888 \\
\hline & $\mathrm{G}$ & 2.845 & 0.119 & 7.407 & 0.533 & 0.920 & 50.072 & 0.884 \\
\hline & $\mathrm{B}$ & 1.088 & 0.208 & 6.845 & 0.811 & 0.745 & 32.471 & 0.883 \\
\hline PCA & $\mathrm{R}$ & 5.819 & 0.084 & 7.701 & 1.176 & 0.963 & 67.427 & 0.866 \\
\hline & $\mathrm{G}$ & 4.113 & 0.123 & 7.602 & 0.958 & 0.960 & 70.296 & 0.821 \\
\hline & & & & & & & & \\
\hline
\end{tabular}

The statistical parameters calculated for fused image with different algorithms and kernel sizes of 3 are given in table 1. Graphical representation of these statistical parameters is shown in fig. 2. Resultant images after fusion are shown in fig. 3 along with original PAN and MS images. In practice, higher spatial resolution is obtained at the cost of spectral resolution and hence correlation [1] that can be seen in table1. The performance of any method is evaluated in terms of statistical parameters, such as spatial correlation ( $\mathrm{sCC}$ ) and spectral correlation (CC) between the original MS and fused MS data, image Difference Index (DI), resultant S/R ratio, NRMSE, entropy and Standard Deviation (SD) of resultant images. Ideally, the $\mathrm{CC}, \mathrm{sCC}, \mathrm{SD}, \mathrm{S} / \mathrm{N}$ ratio, entropy should be high and DI and NRMSE should be low for better performance of the fusion method. From the parameters listed in the table-1, it is seen that the performance of ISH5 method is the best followed by HLS and ISH3 methods when the kernel size of $3 \times 3$ is used. Evaluations of the performance with varying kernel size from 3 to 15 are listed in Table 2 . It can be observed that, the CC as well as sCC, the two most important parameters, decrease with increasing kernel size.

Table2: Statistical parameters derived for ISH5 method of fusion for kernel size adopted in spatial filtering of pan and intensity images

\begin{tabular}{|c|c|c|c|c|c|c|c|}
\hline $\begin{array}{c}\text { Kernel } \\
\text { size }\end{array}$ & SNR & NRMSE & EN & DI×10 & CC & SD & SCC \\
\hline 3 & 5.417 & 0.094 & 7.663 & 2.097 & 0.965 & 69.633 & 0.850 \\
\hline & 4.819 & 0.099 & 7.632 & 0.812 & 0.960 & 66.740 & 0.850 \\
\hline & 4.685 & 0.095 & 7.553 & 0.978 & 0.960 & 65.085 & 0.842 \\
\hline 5 & 5.284 & 0.094 & 7.709 & 0.400 & 0.957 & 67.635 & 0.815 \\
\hline & 4.761 & 0.098 & 7.657 & 0.504 & 0.952 & 64.837 & 0.817 \\
\hline & 4.579 & 0.094 & 7.570 & 0.560 & 0.950 & 63.444 & 0.808 \\
\hline 7 & 4.813 & 0.103 & 7.728 & 0.661 & 0.945 & 66.253 & 0.795 \\
\hline & 4.369 & 0.106 & 7.677 & 0.331 & 0.939 & 63.577 & 0.797 \\
\hline & 4.172 & 0.103 & 7.593 & 0.614 & 0.937 & 62.280 & 0.789 \\
\hline 9 & 4.414 & 0.112 & 7.740 & 0.950 & 0.932 & 64.982 & 0.788 \\
\hline & 4.029 & 0.115 & 7.690 & 0.454 & 0.925 & 62.435 & 0.791 \\
\hline & 3.827 & 0.113 & 7.610 & 0.650 & 0.922 & 61.203 & 0.783 \\
\hline 11 & 4.099 & 0.121 & 7.745 & 0.927 & 0.919 & 63.845 & 0.786 \\
\hline & 3.757 & 0.124 & 7.698 & 0.555 & 0.911 & 61.420 & 0.789 \\
\hline & 3.557 & 0.121 & 7.623 & 0.744 & 0.908 & 60.245 & 0.781 \\
\hline 15 & 3.649 & 0.136 & 7.748 & 0.248 & 0.894 & 62.052 & 0.786 \\
\hline & 3.366 & 0.139 & 7.708 & 0.822 & 0.884 & 59.836 & 0.789 \\
\hline & 3.172 & 0.137 & 7.640 & 0.751 & 0.879 & 58.758 & 0.782 \\
\hline
\end{tabular}

The false colour composites (FCCs) of the fused images for the ten selected methods are shown in fig. 3. Also, the original 


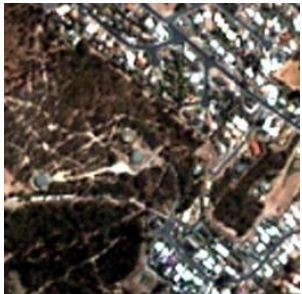

a. MS

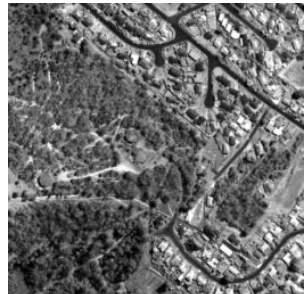

b. PAN

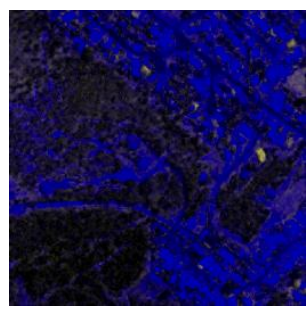

C. HSV

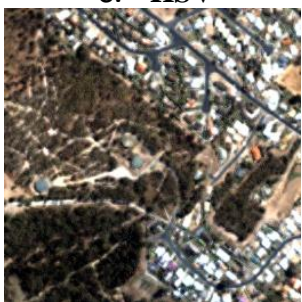

h. IHS5

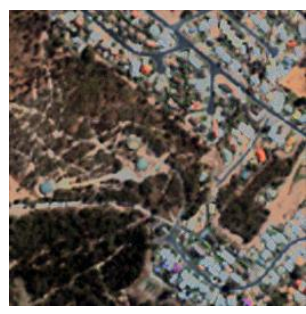

d. IHS1

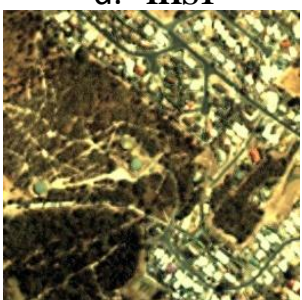

i. IHS6

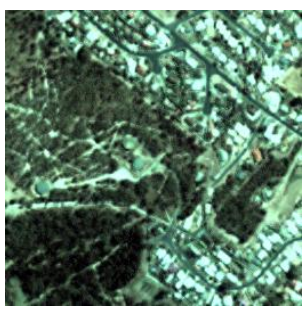

e. IHS2

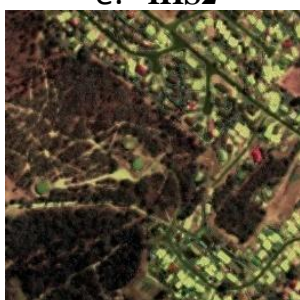

j. IHS7

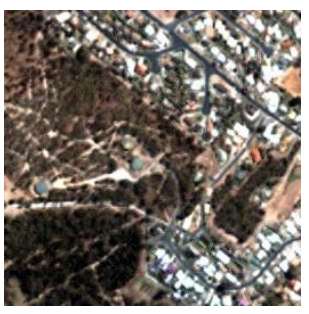

f. IHS3

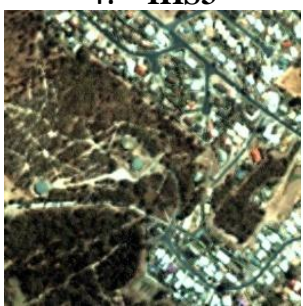

k. PCA

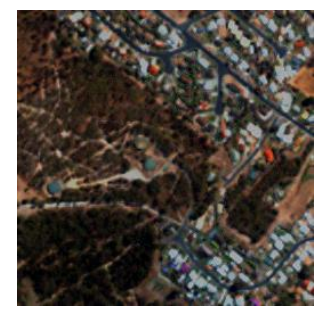

g. IHS4

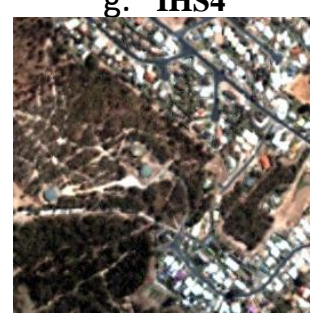

I. HLS

Fig.3 FCC of Fused multispectral images following different methods

PAN and MS images of IKONSOS-2 are shown in the figure for reference. It can be clearly seen that colour-wise the FCCs of IHS5, HLS and IHS3 match quite well with the FCC of the original MS image. The statistical parameters listed in table 1 support this observation quite well. The FCCs of fused images based on the method followed in this paper not only show good spectral matching or colour matching but also show lot of improvement in the spatial features and sharpness of the objects.

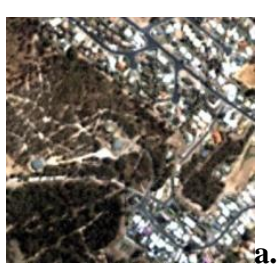

3x3 Kernel

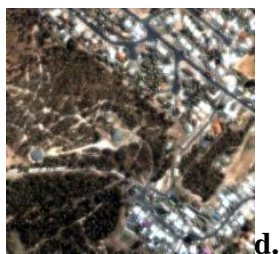

9x9 Kernel

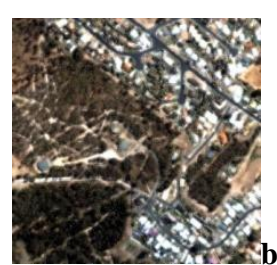

$5 \times 5$ Kernel

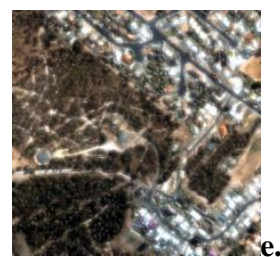

11x11 Kernel

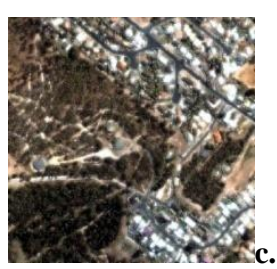

7x7 Kernel

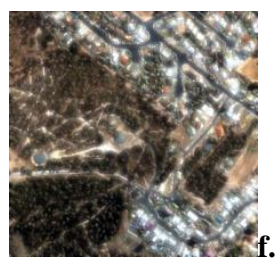

$15 \times 15$ Kernel
Fig.4 FCC of Fused MS images following the best performing method ISH5
With increase in kernel size used for filtering of Pan and intensity images, it is found that the high spatial frequency components are filtered out. The best results are expected with the filtered image using smallest kernel size, i.e. $3 \times 3$ kernels. This is seen very clearly in the fig. 4 . With the increasing kernel size, the features get blurred. This is clearly reflected in the $\mathrm{sCC}$ and $\mathrm{CC}$ parameter values listed in the table 2 .

The table 3 shows the performance of the proposed methodology using the IKONOS-2 image subsets with varying dominant land cover type following the best fusion technique, i.e. the IHS5. The data sets dominating by residential land-cover performance show good results with IHS1 and IHS5 approaches. Open ground cover dominated image gives better performance with IHS3, IHS5 and PCA approaches. The table shows that the spectral correlation coefficient (CC) shows improvement with proposed method albeit at little bit loss of spatial correlation (sCC).

The proposed methodology is also applied to 5 different IKONOS-2 image subsets described above. These sub images exhibit different type of dominant land use/land cover classes. Performance of the proposed technique in case of IKONOS-2 image subsets with varying dominant land cover type (for IHS5 method) is shown in the table 4. According to results of statistical analysis, it is observed that the sub images with large area under vegetation cover, soil cover, and dense urban areas perform well with IHS1, IHS3 and IHS5 approaches. The sub images with sparse urban dominant types perform satisfactory with IHS4, and IHS7 approaches. 
Table 3: Performance of current method in comparison with earlier method published in [1]

\begin{tabular}{|c|l|l|l|l|l|l|l|l|l|l|l|}
\hline Parameter & Method & HSV & IHS1 & IHS2 & IHS3 & IHS4 & IHS5 & IHS6 & HLS & IHS7 & PCA \\
\hline \multirow{3}{*}{ CC } & Earlier & 0.09 & 0.69 & 0.66 & 0.69 & 0.69 & 0.69 & 0.65 & 0.69 & 0.33 & 0.69 \\
\cline { 2 - 12 } & Proposed & 0.29 & 0.93 & 0.95 & 0.96 & 0.96 & 0.96 & 0.95 & 0.96 & 0.87 & 0.96 \\
\hline \multirow{3}{*}{$\mathrm{sCC}$} & Earlier & 0.80 & 0.98 & 0.98 & 0.99 & 0.98 & 0.99 & 0.98 & 0.99 & 0.93 & 0.99 \\
\cline { 2 - 11 } & Proposed & 0.73 & 0.87 & 0.84 & 0.85 & 0.88 & 0.85 & 0.84 & 0.85 & 0.88 & 0.85 \\
\hline
\end{tabular}

Table 4: Performance of the proposed technique in case of IKONOS-2 image subsets with varying dominant land cover type (For IHS5 method)

\begin{tabular}{|c|c|c|c|c|c|c|c|c|c|}
\hline $\begin{array}{l}\text { Image } \\
\text { Segment } \\
\text { SN }\end{array}$ & $\begin{array}{l}\text { Spectral } \\
\text { Band }\end{array}$ & SNR & $\begin{array}{c}\text { NRMS } \\
\quad \text { E }\end{array}$ & EN & $\mathrm{DI} \times 10^{3}$ & $\mathrm{CC}$ & SD & sCC & $\begin{array}{c}\text { Dominant Land cover } \\
\text { Type(s) }\end{array}$ \\
\hline \multirow{3}{*}{266} & $\mathrm{R}$ & 5.749 & 0.040 & 6.141 & 1.848 & 0.958 & 21.945 & 0.932 & \multirow{3}{*}{ Soil } \\
\hline & G & 4.856 & 0.043 & 5.818 & 0.836 & 0.948 & 20.028 & 0.933 & \\
\hline & B & 4.345 & 0.040 & 5.626 & 12.473 & 0.941 & 18.830 & 0.930 & \\
\hline \multirow{3}{*}{177} & $\mathrm{R}$ & 5.041 & 0.054 & 6.508 & 0.940 & 0.951 & 32.411 & 0.921 & \multirow{3}{*}{ Dense urban area } \\
\hline & G & 4.906 & 0.055 & 6.380 & 1.286 & 0.950 & 32.564 & 0.922 & \\
\hline & B & 4.847 & 0.054 & 6.355 & 4.700 & 0.954 & 33.501 & 0.920 & \\
\hline \multirow{3}{*}{150} & $\mathrm{R}$ & 3.702 & 0.052 & 5.914 & 0.219 & 0.916 & 23.953 & 0.917 & \multirow{3}{*}{ Sparse urban area } \\
\hline & G & 3.800 & 0.052 & 5.875 & 0.282 & 0.923 & 25.261 & 0.917 & \\
\hline & B & 3.233 & 0.052 & 5.876 & 6.397 & 0.891 & 21.300 & 0.917 & \\
\hline \multirow{3}{*}{147} & $\mathrm{R}$ & 5.417 & 0.094 & 7.663 & 2.097 & 0.965 & 69.633 & 0.850 & \multirow{3}{*}{$\begin{array}{l}\text { Soil, vegetation } \\
\text { buildings }\end{array}$} \\
\hline & G & 4.819 & 0.099 & 7.632 & 0.812 & 0.960 & 66.740 & 0.850 & \\
\hline & B & 4.685 & 0.095 & 7.553 & 0.978 & 0.960 & 65.085 & 0.842 & \\
\hline \multirow{3}{*}{16} & $\mathrm{R}$ & 6.922 & 0.031 & 5.913 & 0.515 & 0.962 & 19.462 & 0.908 & \multirow{3}{*}{ Soil and vegetation } \\
\hline & G & 5.036 & 0.035 & 5.470 & 0.316 & 0.946 & 16.628 & 0.911 & \\
\hline & B & 5.180 & 0.031 & 5.030 & 0.808 & 0.922 & 13.835 & 0.908 & \\
\hline
\end{tabular}

\section{CONCLUSION}

Image fusion or Pan sharpening methods which give sharpened multispectral images with retention of high spatial frequencies from Pan image and high spectral resolution from MS image are of great value in indirectly obtaining high quality of multispectral images for land use classification and any radiometric analysis. This is a very active filed of research. The present study shows that new technique of merging high spatial frequency component with low frequency component of intensity image and using the merged image in pan-sharpening does improve the retention of both the high spatial resolution features of Pan and high spectral resolution information from MS images. This methodology showed marked improvement in performance by increasing spectral correlation between Pan and fused MS and between original MS and fused MS images, respectively, albeit at some marginal loss in the spatial correlation. The high level of retention of spectral information is especially significant in view of expected better classification accuracy.

\section{ACKNOWLEDGMENT}

Authors would like to thank Director, Bhaskaracharya Institute for Space Applications and Geo-informatics (BISAG), Gandhinagar for providing infrastructure and encouragement. We are also thankful to NASA/GLCF, USA for making the IKONOS data available freely on its web site. 


\section{REFERENCES}

[1] Megha K. Mehta, Nehal G. Chitaliya, Paru Thakkar and Madhukar B. Potdar, "Comparison of Performance of various Image Fusion Techniques using IKONOS-2 data”, International Journal of Soft Computing and Engineering (IJSCE), vol.3, issue 6, January-2014, pp. 232-235.

[2] Hardik Dhamecha, Tanish Zaveri and M. B. Potdar, NDVI Control based High Frequency Injection Multispectral Image Fusion Method, 2012, 978-1-4673-1159-5/12 @2012 IEEE, IGARSS-2, 2012, pp. 3513-3516.

[3] Hardik M. Dhamecha, Tanish Zaveri and Madhukar B. Potdar, A New Algorithm for Multispectral Image Fusion, IEEE-Nuicone-2011.

[4] Miloud Chikr El-Mezouar, Nasreddine Taleb, Kidiyo Kpalma and Joseph Ronsin, "An IHS-Based Fusion for Color Distortion Reduction and Vegetation Enhancement in IKONOS Imagery", IEEE Transactions On Geoscience and Remote Sensing, Vol. 49, No. 5, May 2011, pp. 1590-1602.

[5] Firouz Abdullah Al-Wassai, N.V. Kalyankar, Ali A. AlZuky, "The IHS Transformations Based Image Fusion", Computer Vision and Pattern Recognition (cs. CV), July 2011, pp. 1107-3348.

[6] Jaewan Choi, Kiyun Yu, and Yongil Kim, "A New Adaptive Component-Substitution-Based Satellite Image Fusion by Using Partial Replacement", IEEE Transactions On Geoscience And Remote Sensing, Vol. 49, No. 1, January 2011, pp. 295-309.

[7] Jose A. Malpica, "Hue Adjustment to IHS Pan-Sharpened IKONOS Imagery for Vegetation Enhancement", IEEE Geoscience And Remote Sensing Letters, Vol. 4, No. 1, January 2007, pp.27-31.
[8] V.P.S. Naidu and J.R. Raol, "Pixel-level Image Fusion using Wavelets and Principal Component Analysis", Defence Science Journal, Vol. 58, No. 3, May 2008, pp. 338-352.

[9] Zhijun Wang, Djemel Ziou, Costas Armenakis, Deren Li, and Qingquan Li, "A Comparative Analysis of Image Fusion Methods", IEEE Transactions On Geoscience And Remote Sensing, Vol. 43, No. 6, June 2005, pp. 1391-1402.

[10] Myungjin Choi, “A New Intensity-Hue-Saturation Fusion Approach to Image Fusion With a Tradeoff Parameter", IEEE Transactions On Geoscience And Remote Sensing, Vol. 44, No. 6, June 2006, pp. 1672-1682.

[11] Bruno Aiazzi, Stefano Baronti, Massimo Selva, "Improving Component Substitution Pansharpening Through Multivariate Regression of MS+Pan Data", IEEE Geoscience And Remote Sensing, Vol. 45, No. 10, October 2007, pp. 3230-3239.

[12] Kazi A. Kalpoma and Jun-ichi Kudoh, "Image Fusion Processing for IKONOS 1-m Color Imagery", IEEE Geoscience And Remote Sensing, Vol. 45, No. 10, October 2007, pp. 3075-3086.

[13] T. Tu, S. Su, H. Shyu, and P. S. Huang, "A new look at his-like image fusion methods", Inf. Fusion, vol. 2, April 2001, pp. 177-186.

[14] Li S., Kwok J. T., Wang Y., "Using the Discrete Wavelet Frame Transform To Merge Landsat TM And SPOT Panchromatic Images", Information Fusion 3, 2002, pp.17-23

[15] Hsu S. L., Gau P.W., Wu I L., and Jeng J.H., "RegionBased Image Fusion with Artificial Neural Network", World Academy of Science, Engineering and $\begin{array}{lllll}\text { Technology, } & 53, \quad 2009, & \text { pp } & 156 & -159\end{array}$ 